\title{
3D Electron Diffraction for Structure Analysis of Challenging Inorganic Materials
}

Mariana Klementová, Lukas Palatinus and Paul Klar

FZU - Institute of Physics of the Czech Academy of Sciences, Prague, Hlavni mesto Praha, Czech Republic

3D electron diffraction (3DED) covers a large number of methods unified by a common goal - structure determination from electron diffraction experiments in a similar way as it is done by single crystal X-ray diffraction. The method was first introduced by Kolb et al. in 2007 [1], and since then it has acquired a number of different flavors [2]. 3DED has been used for solving a wide variety of crystal structures covering oxides, silicates, intermetallics, metal-organic frameworks, small organic molecule structures and proteins [3].

Electron diffraction is a powerful technique for the study of nanomaterials. Because of the strong interaction of electrons with matter, diffraction from individual nanocrystals can be exploited for structure solution and differences among individual nanocrystals within a sample can be studied. However, the strong interaction also leads to multiple diffraction of electrons and violations of the kinematical approximation. Therefore, electron diffraction data require a dedicated data reduction and especially refinement. Our group at FZU now offers a complete software support for 3DED structural analysis including the analysis of modulated structures.

The PETS software for the processing of electron diffraction data [4] offers a number of features unique to electron diffraction such as treatment of precession electron diffraction data. In addition to dealing with periodic structures, the data processing of modulated structures is also available including the definition of modulation wave vectors and data integration. For the structure solution, the program Superflip has been long available for X-rays [5] but it can be also applied to 3DED data. Jana2006 software is then well known as a versatile and very powerful tool for the analysis of challenging crystal structures, and it now fully supports structure analysis from 3DED data, including advanced features as the combination of several datasets from different crystals or refinement against data from twinned crystals [6]. Last but not least, the structures, including incommensurately modulated structures, can be refined by the dynamical approach using the program Dyngo [7]. This approach uses the full dynamical diffraction theory in the calculation of model intensities and provides refinement quality and accuracy of structure models that is superior to other approaches in 3DED. Several examples will be presented to illustrate the above described procedure.

One of the ultimate challenges in the crystal structure determination is the localization of hydrogen atoms. Hydrogen atoms, with only one electron, have very low scattering power for X-rays and electrons, and they often have large atomic vibrations, which makes them difficult to detect. The localization of hydrogen in nanocrystals revealed by PEDT using dynamical refinement an inorganic zeotype material (microporous cobalt-aluminophosphate) will be presented (Fig. 1) [8].

In the structure determination of $\mathrm{Ni}_{8} \mathrm{Ti}_{5}$, the assignment of atomic types due to the proximity of scattering powers of Ti and Ni was solved by several approaches: (1) analysis of the Wyckoff position multiplicities, (2) refinement of displacement parameters, and (3) geometrical analysis of the structure and comparison with known structures. These approaches consistently pointed to one distribution, which was confirmed by a significant improvement of the refinement figures of merit (Fig. 1). The dynamical refinement turned 
out to be an indispensable technique that provided the necessary sensitivity to allow the full determination of the structure [9].

Another challenging example is the case of 5/2-mullite $\left(\mathrm{Al}_{4+2} \mathrm{Si}_{2-2} \mathrm{O}_{10-x}, x \approx 0.5\right)$. Its modulated structure is not accessible by powder diffraction because the satellite reflections are extremely weak and the crystallites are too small for single crystal XRD. 3DED and dynamical refinement taking first and second order satellite reflections into account allows to determine accurate modulation functions to understand the aperiodic structure based on vacancy and Al/Si ordering (Fig. 1) [10, 11].
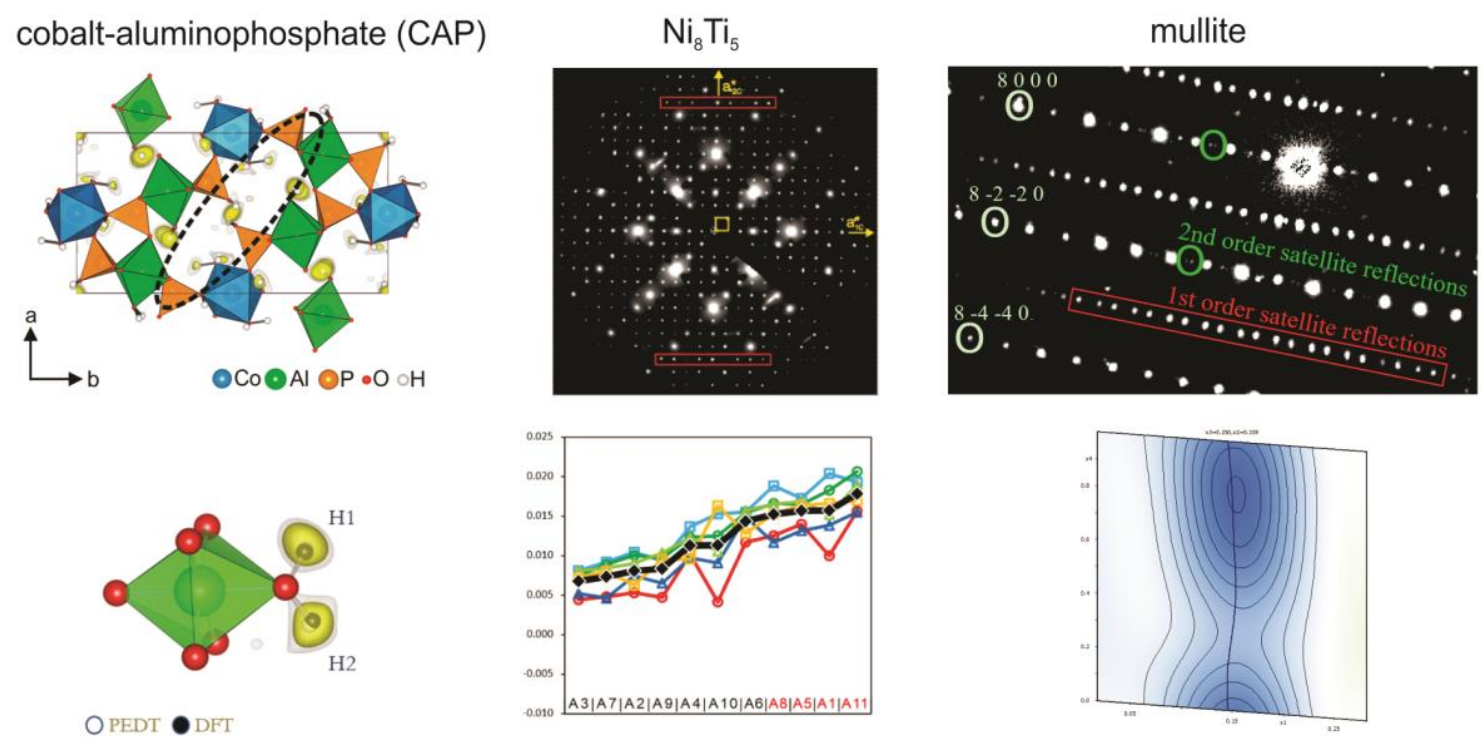

Figure 1. CAP: (top) Structure with a superimposed difference potential map showing maxima at the positions of the hydrogen atoms, (bottom) Isosurface representation of the difference potential map for Al-octahedron.Ni8Ti5: (top) Section through experimental ED data, (bottom) Atomic displacement parameters for all atoms assigned as $\mathrm{Ni}$; sites A8/A5/A1/A11 should be occupied by Ti.Mullite: (top) Diffraction pattern of the [0-11] zone axis. First and second order satellite reflections are marked in red and green, respectively. (bottom) Superspace section through the electrostatic potential of the tetrahedral site indicating the occupational modulation and weak displacive modulation.

\section{References}

[1] U. Kolb et al., Ultramicroscopy 107 (2007) p.507.

[2] M. Gemmi and A.E. Lanza, Acta Cryst. B75 (2019) p. 495.

[3] M. Gemmi et al., ACS Cent. Sci. 5 (2019) p.1315.

[4] L. Palatinus et al., Acta Cryst. B75 (2019) p 512.

[5] L. Palatinus and G. Chapuis, J. Appl. Cryst. 40 (2007) p 786.

[6] V. Petricek, M. Dusek, L. Platinus, Z. Krist 229-5 (2014) p. 345.

[7] L. Palatinus, V. Petricek, C.A. Correa, Acta Crystallogr., Sect. A: Found. Adv.71 (2015) p. 235.

[8] L. Palatinus et al., Science 355 (2017) p.166.

[9] M. Klementova et al., Intermetallics 85 (2017) p. 110.

[10] P.B. Klar, I. Etxebarria, G. Madariaga, IUCrJ 5 (2018) p. 497

[11] The authors acknowledge funding from the Czech Science Foundation (Project 19-08032S) and support by the project No. LO1603 under the Ministry of Education, Youth and Sports National sustainability programme I of Czech Republic. 